Towards a more robust and replicable science of infant development Michael C. Frank

Department of Psychology, Stanford University

Please address correspondence to: Michael C. Frank, 450 Serra Mall, Stanford, CA 94305.mcfrank@stanford.edu. 


\section{Towards a more robust and replicable science of infant development}

If you scroll through the table of contents of Infant Behavior and Development ten years

from now, what mix of articles should you find? If the past predicts the future, most articles will be empirical reports: one or a series of experiments conducted at a single site, typically investigating a novel phenomenon or with a new take on a previous experimental paradigm. On the other hand, based on recent changes in psychology more generally, we might expect a broader range of articles. Some might still be new empirical reports from a single laboratory; but others might be larger-scale collaborative studies; still others will be confirmatory replication research; and others will be new methodological and statistical innovations.

These changes have been triggered by a host of different factors, but two threads stand out. The first was a recognition that many of the analytic practices followed by psychologists could substantially inflate the probability of a spurious finding being reported (Simmons, Nelson, \& Simonsohn, 2011). The second was initial experimental evidence for the low rate of replicability - finding the same conclusions from a new dataset - in a sample of high-profile psychology findings (Open Science Collaboration, 2015). These two initial threads have become part of a rich fabric of discussion surrounding the nature of scientific practices and their consequences for the robustness of the research literature (Munafò et al., 2017).

This conversation, sometimes referred to as the "open science movement," has led to both a re-evaluation of a host of specific empirical findings in psychology as well as a broader examination of statistical and methodological assumptions that underpin our research practice. One trend that defines this period is a new emphasis on the importance of direct replication of research findings (Zwaan, Etz, Lucas, \& Donnellan, 2018). Another is a focus on methodological 
and statistical innovations that attempt to increase the efficiency and robustness of novel research in psychology (Shrout \& Rogers, 2018). But the penetrance of these discussions into different research communities in psychology has been far from uniform. While some sub-disciplines - social psychology, for example - have been consumed by these issues, others have been less directly affected.

Developmental psychology - and specifically, infancy research - is an interesting case. As Davis-Keane and Ellis (Update citation: Davis-Keane) review, experimental research focusing on infants and young children features many of the risk factors for low replicability that have been found in other work (Bergmann et al., 2018), including small sample sizes, analytic flexibility, and measures with limited reliability (Button et al., 2013; Fried \& Flake, 2018; Munafò et al., 2017; Simmons et al., 2011). Yet direct replication has been relatively uncommon in this literature, even if some parts of the community have focused on triangulating constructs of interest through conceptual replications (as illustrated by Kucker et al., 2019 in this special issue). Further, developmental research tends to be performed with convenience samples in predominantly high socio-economic status, Western contexts, substantially limiting the robustness and generalizability of our findings (Nielsen, Haun, Kärtner, \& Legare, 2017).

In part due to these concerns, a group of infancy researchers has come together under the moniker "ManyBabies" (Frank et al., 2017). The goal of the group has been to create a collaborative network for conducting best-practices replications of prominent phenomena in infancy research, while fostering increasing awareness of open-science issues within the developmental research community. Many of us have found these discussions to be among the most stimulating parts of our scientific careers. 
As one of the outreach activities of the ManyBabies group, Rebecca Lundwall, Elika Bergelson, and I organized a pre-conference at the 2017 Meeting of the Cognitive Development Society in Portland, Oregon. This meeting featured a host of exciting research that pushed the methodological and theoretical boundaries of the field, leading us to consider - with a generous invitation from Martha Arterberry - what a collection of this research would look like, in the form of a special issue of Infant Behavior and Development. We issued an open call for submissions in spring of 2018, and I agreed to act as action editor for these. The exciting collection of papers you are (likely) now viewing is the result. In my view, they serve as an excellent template for what a sampling of infancy research might look like in the era of open science, being far broader in their approaches than the single lab, multi-experiment package that is still the norm in developmental psychology.

First, research in the era of open science needs to be sensitive to the incentives that govern scientific careers. The perception is often that replication research is less innovative and less valued than original research (Zwaan et al., 2018). Accordingly, scientists respond to this incentive by privileging theoretical novelty (rather than robustness or incremental progress) in their allocation of resources. In this issue, Lundwall (2019) offers a potential vision of how institutional departments could help mitigate the career challenges of following a path towards more open and replicable research.

Second, the papers in the special issue show an admirable emphasis on rigorous methods and methodological innovation. A causal chain connects research that is difficult and expensive to conduct to findings that are less robust: the more expensive work is to do, the less data researchers tend to collect in each sample (raising the probability of spurious findings), and the 
less direct replication is done. Put simply, cheaper data - in terms of time and money - enables more robust science. Thus, the major focus of the methodological work described by papers in the special issue is increasing the efficiency of infancy research.

For example, Schott, Rhemtulla, and Byers-Heinlein (2019) offer a guide to maximizing researchers' ability to use limited samples via the use of conditional stopping - where researchers safely "peek" at data to decide whether to continue testing or stop. Such a practice could allow researchers to make a statistically sound inference to increase a sample size so as to have a higher probability of obtaining a significant result. Data analysis can also be planned to increase the utility of the data, given a particular sample. Addressing this challenge, de Klerk, Wijnen, and de Bree (2019) describe the use of hierarchical Bayesian models to increase the accuracy of assessments of individual infants' abilities.

In a similar vein, addressing the bottleneck of annotating infant looking data by hand, Chouinard, Scott, and Cusack (2019) test the use of computer vision methods. Such methods are an especially promising avenue in light of recent successes in testing infants through online platforms (Scott \& Schulz, 2017; Tran, Cabral, Patel, \& Cusack, 2017). And Kominksy (2019) provides a software platform for presenting stimuli to infants and sharing implementations of infancy paradigms, increasing the ease of repeating the same experiment in different contexts.

Where could all of this automation take us? Wood and Wood (2019) provide a sketch of what the end-point might look like - a vastly more robust and data-rich ecosystem that provides opportunities to look at developmental change on the micro as well as macro scale. Of course, this vision is currently realized with baby chicks, rather than baby humans, but the possibility is 
exciting (and the findings with chicks of course also contribute to the enterprise of understanding human development).

Third, papers in the special issue model best practices in developmental replication. One explicit goal of this special issue was to provide a forum for the publication of systematic and carefully-conducted replication research, and we received a number of high-quality submissions of this type. Interestingly, all four of the replications in the special issue are in the area of social cognitive development, an area that has been the subject of both intense theoretical debate (Baillargeon, Scott, \& He, 2010; Heyes, 2014) and a locus of recent replication activity (Sabbagh $\&$ Paulus, 2018). One of the papers in the special issue examines the importance of different kinds of social cues in infants' encoding of event structure (Silverstein, Gliga, Westermann, \& Parise, 2019). Another, a highly systematic set of studies - featuring six experiments with eight distinct samples - focuses on the phenomenon of action anticipation (UPDATE CITE:

Ganglmayer et al.). A further two studies address phenomena related to false-belief understanding (Dörrenberg, Wenzel, Proft, Rakoczy, \& Liszkowski, 2019; Grosso, Schuwerk, Kaltefleiter, \& Sodian, 2019). And finally, a fifth synthesizes literature related to early false-belief understanding and offers positive suggestions for increasing the robustness of this controversial literature (Rubio-Fernández, 2019). Together these papers model careful replication efforts; although none will be the definitive word on their particular phenomenon of interest, each represents a very substantial effort that will contribute to our understanding of a phenomenon.

One final theme that comes out nicely in several papers of the special issue is that of collaboration. Due to the high costs and logistical challenges of collecting developmental 
samples, our community needs to work together to produce robust and representative scientific findings (Frank et al., 2017). Larger groups of course can gather larger samples. But the advantages of collaboration go well beyond this obvious one. As Ganglmayer et al. (UPDATE CITE) show in their work, multi-lab collaborations can better evaluate multiple different instantiations of an experimental paradigm - producing inferences that do not depend as tightly on the specifics of one method. And for even larger studies like EuroSibs (Jones et al., 2019), the pressure to "get it right" can lead to far more attention to developing reliable and valid measures than is typical in a one-off single-lab study. I am hopeful that it will become far more common in infant research to see multi-lab collaborations that are sparked by the need to gather appropriate sample sizes, to develop more general measures, and to draw more representative conclusions.

In their sum, the papers in this special issue provide a compelling template for the kind of careful empirical work combined with methodological innovation that I hope will increasingly characterize the cutting edge of infancy research. There will always be a place for new paradigms that drive theoretical innovations. At the same time, there needs to be a mix of research in our journals, such that innovations are included alongside replication research and methodological development. Each of these - together with novel experimental work - will make up a part of a more robust and cumulative science of early development. 


\section{References}

Baillargeon, R., Scott, R. M., \& He, Z. (2010). False-belief understanding in infants. Trends in Cognitive Sciences, 14(3), 110-118.

Bergmann, C., Tsuji, S., Piccinini, P. E., Lewis, M. L., Braginsky, M., Frank, M. C., \& Cristia, A. (2018). Promoting replicability in developmental research through meta-analyses: Insights from language acquisition research. Child Development, 89(6), 1996-2009.

Button, K. S., Ioannidis, J. P., Mokrysz, C., Nosek, B. A., Flint, J., Robinson, E. S., \& Munafò, M. R. (2013). Power failure: Why small sample size undermines the reliability of neuroscience. Nature Reviews Neuroscience, 14(5), 365.

Chouinard, B., Scott, K., \& Cusack, R. (2019). Using automatic face analysis to score infant behaviour from video collected online. Infant Behavior and Development, 54, 1-12. https://doi.org/10.1016/j.infbeh.2018.11.004

de Klerk, M., Veen, D., Wijnen, F., \& de Bree, E. (2019). A step forward: Bayesian hierarchical modelling as a tool in assessment of individual discrimination performance. Infant Behavior and Development, 54, XYZ.

Dörrenberg, S., Wenzel, L., Proft, M., Rakoczy, H., \& Liszkowski, U. (2019). Reliability and generalizability of an acted-out false belief task in 3-year-olds. Infant Behavior and Development, 54, 13-21. https://doi.org/10.1016/j.infbeh.2018.11.005

Frank, M. C., Bergelson, E., Bergmann, C., Cristia, A., Floccia, C., Gervain, J., ... Levelt, C. (2017). A collaborative approach to infant research: Promoting reproducibility, best practices, and theory-building. Infancy, 22(4), 421-435.

Fried, E. I., \& Flake, J. K. (2018). Measurement matters. APS Observer, 31(3). 
Grosso, S. S., Schuwerk, T., Kaltefleiter, L. J., \& Sodian, B. (2019). 33-month-old children succeed in a false belief task with reduced processing demands: A replication of Setoh et al. (2016). Infant Behavior and Development, 54, 151-155. https://doi.org/10.1016/j.infbeh.2018.09.012

Heyes, C. (2014). False belief in infancy: A fresh look. Developmental Science, 17(5), 647-659. Jones, E. J. H., Mason, L., Begum Ali, J., van den Boomen, C., Braukmann, R., Cauvet, E., ... Johnson, M. H. (2019). Eurosibs: Towards robust measurement of infant neurocognitive predictors of autism across Europe. Infant Behavior and Development, 57, 101316. https://doi.org/10.1016/j.infbeh.2019.03.007

Kominsky, J. F. (2019). PyHab: Open-source real time infant gaze coding and stimulus presentation software. Infant Behavior and Development, 54, 114-119. https://doi.org/10.1016/j.infbeh.2018.11.006

Kucker, S. C., Samuelson, L. K., Perry, L. K., Yoshida, H., Colunga, E., Lorenz, M. G., \& Smith, L. B. (2019). Reproducibility and a unifying explanation: Lessons from the shape bias. Infant Behavior and Development, 54, 156-165. https://doi.org/10.1016/j.infbeh.2018.09.011

Lundwall, R. A. (2019). Changing institutional incentives to foster sound scientific practices: One department. Infant Behavior and Development, 55, 69-76. https://doi.org/10.1016/j.infbeh.2019.03.006

Munafò, M. R., Nosek, B. A., Bishop, D. V., Button, K. S., Chambers, C. D., Du Sert, N. P., ... Ioannidis, J. P. (2017). A manifesto for reproducible science. Nature Human Behaviour, l(1), 0021. 
Nielsen, M., Haun, D., Kärtner, J., \& Legare, C. H. (2017). The persistent sampling bias in developmental psychology: A call to action. Journal of Experimental Child Psychology, $162,31-38$.

Open Science Collaboration. (2015). Estimating the reproducibility of psychological science. Science, 349(6251), aac4716.

Rubio-Fernández, P. (2019). Publication standards in infancy research: Three ways to make Violation-of-Expectation studies more reliable. Infant Behavior and Development, 54, 177-188. https://doi.org/10.1016/j.infbeh.2018.09.009

Sabbagh, M. A., \& Paulus, M. (2018). Replication studies of implicit false belief with infants and toddlers. Cognitive Development, 46, 1-3. https://doi.org/10.1016/j.cogdev.2018.07.003

Schott, E., Rhemtulla, M., \& Byers-Heinlein, K. (2019). Should I test more babies? Solutions for transparent data peeking. Infant Behavior and Development, 54, 166-176. https://doi.org/10.1016/j.infbeh.2018.09.010

Scott, K., \& Schulz, L. (2017). Lookit (part 1): A new online platform for developmental research. Open Mind, 1(1), 4-14.

Shrout, P. E., \& Rodgers, J. L. (2018). Psychology, science, and knowledge construction: Broadening perspectives from the replication crisis. Annual review of psychology, 69, 487-510.

Silverstein, P., Gliga, T., Westermann, G., \& Parise, E. (2019). Probing communication-induced memory biases in preverbal infants: Two replication attempts of Yoon, Johnson and Csibra (2008). Infant Behavior and Development, 55, 77-87. https://doi.org/10.1016/j.infbeh.2019.03.005 
Simmons, J. P., Nelson, L. D., \& Simonsohn, U. (2011). False-positive psychology: Undisclosed flexibility in data collection and analysis allows presenting anything as significant. Psychological Science, 22(11), 1359-1366.

Tran, M., Cabral, L., Patel, R., \& Cusack, R. (2017). Online recruitment and testing of infants with Mechanical Turk. Journal of Experimental Child Psychology, 156, 168-178.

Wood, S. M. W., \& Wood, J. N. (2019). Using automation to combat the replication crisis: A case study from controlled-rearing studies of newborn chicks. Infant Behavior and Development, 57, 101329. https://doi.org/10.1016/j.infbeh.2019.101329

Zwaan, R. A., Etz, A., Lucas, R. E., \& Donnellan, M. B. (2018). Making replication mainstream. Behavioral and Brain Sciences, 41. 